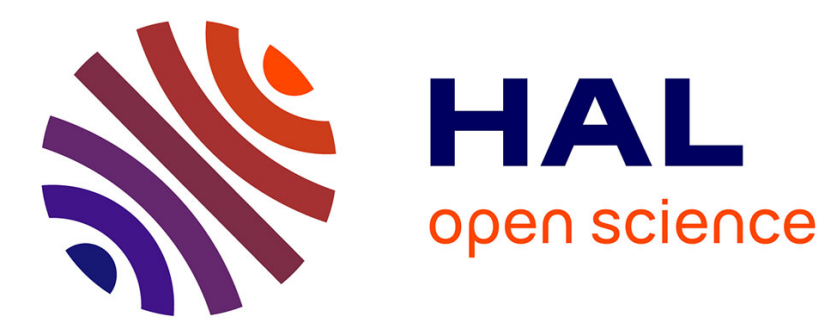

\title{
On the multi-modal sensing of electrical arcs
}

Costin Vasile, Cornel Ioana

\section{To cite this version:}

Costin Vasile, Cornel Ioana. On the multi-modal sensing of electrical arcs. SAS 2017 - Sensors Applications Symposium, Mar 2017, Glassboro, United States. pp.1 - 5, 10.1109/SAS.2017.7894117 . hal-01592664

\section{HAL Id: hal-01592664 https://hal.science/hal-01592664}

Submitted on 25 Sep 2017

HAL is a multi-disciplinary open access archive for the deposit and dissemination of scientific research documents, whether they are published or not. The documents may come from teaching and research institutions in France or abroad, or from public or private research centers.
L'archive ouverte pluridisciplinaire HAL, est destinée au dépôt et à la diffusion de documents scientifiques de niveau recherche, publiés ou non, émanant des établissements d'enseignement et de recherche français ou étrangers, des laboratoires publics ou privés. 


\section{On the Multi-Modal Sensing of Electrical Arcs}

\author{
Costin Vasile \\ Gipsa-lab, University Grenoble-Alpes \\ Grenoble, France \\ costin.vasile@gipsa-lab.grenoble-inp.fr
}

\author{
Cornel Ioana \\ Gipsa-lab, University Grenoble-Alpes \\ Grenoble, France \\ cornel.ioana@gipsa-lab.grenoble-inp.fr
}

\begin{abstract}
Electrical arc faults represent a danger for today's power generation systems, and their detection can have a serious positive economic impact. In this paper we present a system for the detection and localization of DC electrical arc faults (typical for a photovoltaic power generation system). Our approach is based on the multidimensionality of the arc fault emissions, therefore we use a dual sensing system, composed by electromagnetic \& acoustic sensors. Also, we present a comparison between two possible signal processing methods in order to recover the TOA ("time of arrival"), which then enables us to precisely localize the source of the arc fault.
\end{abstract}

Keywords - electrical arc fault; acoustic; electromagnetic; time of arrival; transient detection.

\section{INTRODUCTION}

The increasing amount of energy production and of the scale of energy producing facilities increase the wear of these installations. Then, the surveillance systems need to be defined in order to avoid fault generation and propagation. One such case is the occurrence of electrical arc faults in photovoltaic (PV) systems. The physical impact of such a fault can be seen in Fig. 1.

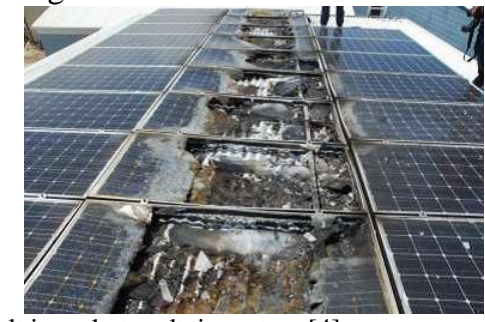

Fig. 1 DC arc fault in a photovoltaic system [4].

Our system exploits the multiple emissions of the arc fault phenomenon, by measuring the generated electromagnetic and acoustic waves. With these measurements, the system (with the help of different signal processing methods) succeeds in detecting the presence of the arc fault, as well as computing the position of its source.

In the first section, we will give a short description of the system (with regards to the sensors used and their positioning). In section II, we will describe two different signal processing methods with the aim of detecting and localizing the arc fault. In section III, results and performances for one type of measurement will be evaluated, in order to conclude what method works better in this context and to present our explanation for the results.

\section{SYSTEM DESCRIPTION}

The study of electrical arc faults is a topic of interest in the scientific community [1]-[3], as well as in the field of detecting DC electrical arc faults that occur in PV systems.

The authors have also previously exploited the arc fault feature of generating electromagnetic and acoustic waves, as can be seen in [5]-[7]. In this paper we will present a more up to date embodiment of the system, with two classical TOA estimation methods, which are generally used for transient detection.

The physical feature that makes the system robust is that the generation of electromagnetic waves is instantaneous (to all extents and purposes) and serves as an external trigger for the acoustic sensing channels. By having the reference $t_{0}$ of the arc fault ignition given by the electromagnetic sensor, we then proceed to calculate the TOA on each of three acoustic sensing channels. Once having done this, by employing the difference of TOA ("difference in time of arrival"-DTOA) between each of the three channels, we manage to compute an estimation of the position of the arc fault source. Fig. 2 shows the elements that were used for this system.

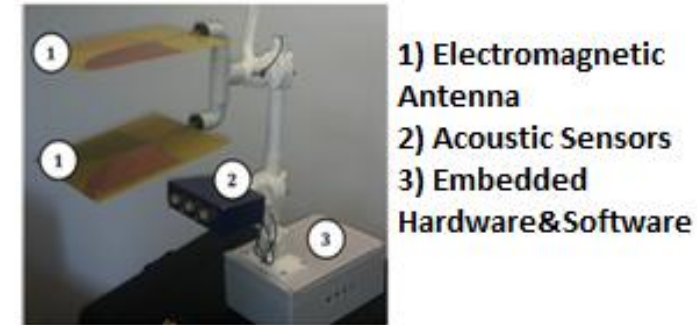

Fig. 2 Description of the detection and localization system.

The electromagnetic sensor consists of two FR-4 double sided antennas (element 1 of Fig. 2). The acoustic sensing is done with three ultrasound microphones, with a frequency response which makes them acceptable for use in the 10$135 \mathrm{kHz}$ range.

An example of a measurement is in Fig. 3, where we can clearly see the arc fault ignition on the electromagnetic sensor and the acoustic sensor reception, which arrives at a slightly different TOA on each channel. Again, the first sharp peak in the electromagnetic signal (blue) triggers the data acquisition for the acoustic sensing. 


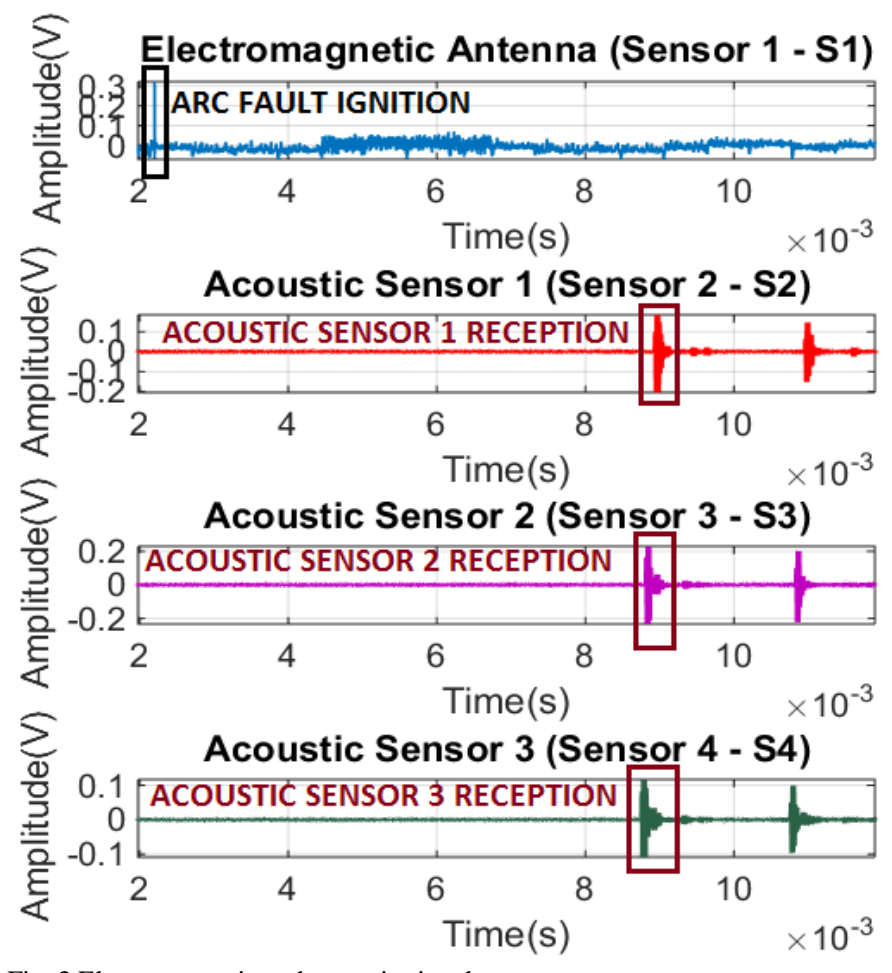

Fig. 3 Electromagnetic and acoustic signals.

Once having computed the three TOA's, we then solve the following equation system in order to complete the localization [5]-[7]:

$$
\left\{\begin{array}{l}
d_{P S_{3}}-d_{P S_{2}}=v \times t_{32} \\
d_{P S_{4}}-d_{P S_{3}}=v \times t_{43} \\
d_{P S_{4}}-d_{P S_{2}}=v \times t_{42}
\end{array}\right.
$$

, where $d_{P S_{i}}=\sqrt{\left(x_{S_{i}}-x_{P}\right)^{2}+\left(y_{S_{i}}-y_{P}\right)^{2}+\left(z_{S_{i}}-z_{P}\right)^{2}}$, $t_{(i+1) i}=t_{i+1}-t_{i}$ is the difference of time of arrival (DTOA), $\mathbf{t}_{\mathrm{i}}$ is the time of arrival at each acoustic sensor $\mathbf{S}_{\mathrm{i}}$, $S_{i}\left(x_{S_{i}}, y_{S_{i}}, z_{S_{i}}\right), v$ is the speed of sound and $P\left(x_{\mathrm{P}}, y_{\mathrm{P}}, z_{\mathrm{P}}\right)$ is the photovoltaic panel location (the arc fault source). A visual description of the measurements setup is presented in Fig. 4 (antenna - S1, acoustic sensor $1-\mathrm{S} 2$, acoustic sensor $2-\mathrm{S} 3$, acoustic sensor $3-\mathrm{S} 4$, consistent with Fig. 3) .

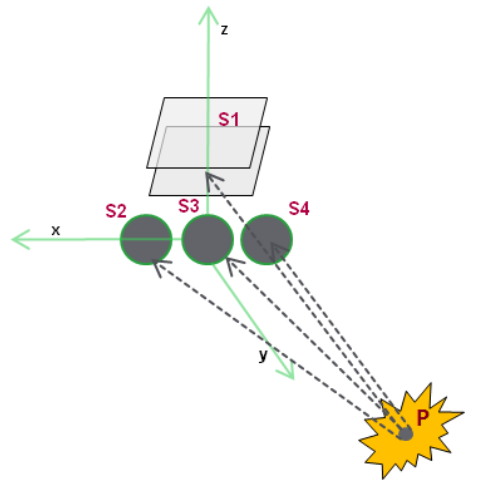

Fig. 4. Experimental measurement setup description.

\section{Signal Processing Methods}

In this study, we present two classical methods in transient signal detection in order to detect the sharp peaks in each of the signals in Fig. 3.

The two approaches are:

- Pre-filtering and finding the time instant with the maximal energy (energy detector);

- Sliding window kurtosis and standard deviation analysis.

\section{A. Energy detector}

Firstly a time-frequency analysis (spectrogram) of the signals is performed. The aim is to observe how the frequency content of the signals is spread in the $20-70 \mathrm{kHz}$ range (Fig. 5), so we applied an appropriate pass-band filter in order to denoise the signal.

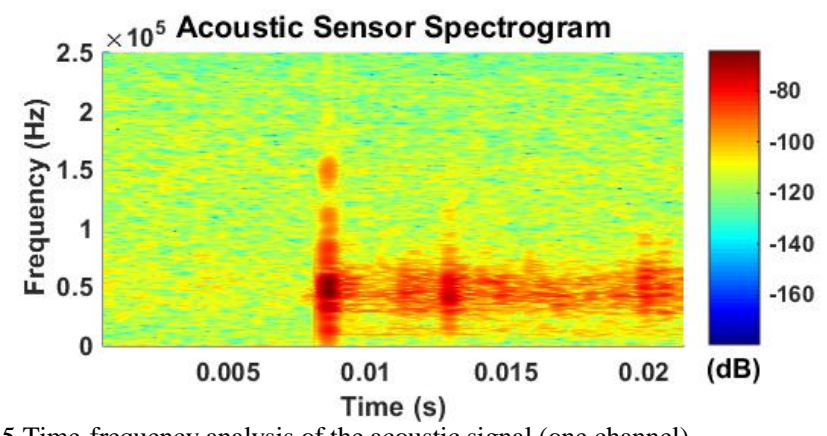

Fig. 5 Time-frequency analysis of the acoustic signal (one channel).

On this denoised signal the well known energy detector is applied, as depicted in Fig. 6.

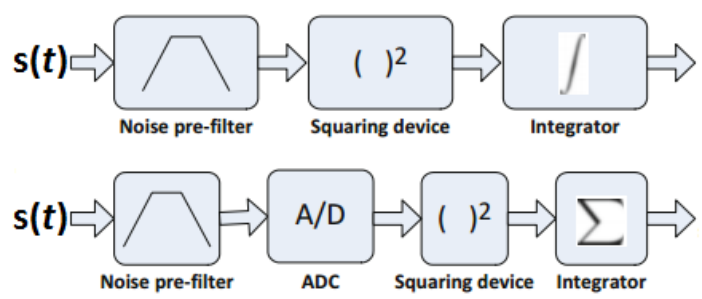

Fig. 6 Continuous (analog) energy detector (Upper); Discrete (numerical) energy detector.

The basic principle of this approach is that it measures the energy associated with the received signal over a specified time duration (and in this case, also in a specified bandwidth). The measured value is compared afterwards with an appropriately selected threshold to determine the presence or the absence of the transient signal [8], cases which define the binary hypothesis testing problem [10]:

\section{\{ Hypothesis $0\left(H_{O}\right)$ : signal (arc fault)is absent. Hypothesis $1\left(H_{1}\right)$ : signal (arc fault)is present.}

Equation 2 translates in the following formulation:

$$
y(n)= \begin{cases}w(n) \quad: H_{0} \\ x(n)+w(n): H_{1}\end{cases}
$$

where $x(n)$ is the signal to be detected (the arc fault) and $w(n)$ is the background noise, with known variance $2 \sigma_{w}^{2}$. 
The associated test statistic, in a numerical implementation, after appropriate pre-filtering, sampling, squaring and integration is given in Equation 4:

$$
\Lambda=\sum_{n=1}^{N}|y(n)|^{2}
$$

where $N$ is the window length (which will be appropriately chosen for our detection purpose).

In the context of this formulation of our detection problem, the probability distributions of each of the two hypotheses will be computed based on the carried out measurements, which will then yield the metrics on which we will base our final evaluation of the proposed solutions: the probability of detection (probability of correctly detecting $H_{l}$ ) and probability of false alarms (probability of incorrectly detecting $H_{0}$ as $H_{l}$ ).

In conclusion, it is a classical detection scheme and in the case of our application, with the type of signals depicted in Fig. 3 , it is advisable to test its performances with regards to our arc fault detection objective.

\section{B. Sliding window kurtosis and standard deviation analysis}

For this approach, instead of analyzing the output of an integrator (such as the case for the energy detector), we consider the standard deviation and the kurtosis values of the samples that are located in the sliding window of analysis. The variation of statistical moments (in this case, time variation) can be a good indicator for transient signal detection.

The standard deviation of a random variable $X$, as a statistical moment, is defined in eq. 5 :

$$
\sigma=\sqrt{E\left[X^{2}\right]-(E[X])^{2}}
$$

The unbiased estimator of the sample standard deviation is given in eq. 6 , for a signal $x$ of $N$ samples and mean $\bar{x}$ :

$$
\sigma=\sqrt{\frac{1}{N-1} \sum_{i=1}^{N}\left(x_{i}-\bar{x}\right)^{2}}
$$

The kurtosis of a random variable $X$ (with mean $\mu$ ) is the fourth standardized moment, as defined in eq. 7:

$$
\operatorname{Kurt}[X]=\frac{E\left[(X-\mu)^{4}\right]}{\left(E\left[(X-\mu)^{2}\right]\right)^{2}}
$$

The sample kurtosis formula that was used for this work (for $N$ samples, with sample mean $\bar{x}$ ) is given in eq. 8:

$$
\operatorname{Kurt}[X]=\frac{\frac{1}{N} \sum_{i=1}^{N}\left(x_{i}-\bar{x}\right)^{4}}{\left(\frac{1}{N} \sum_{i=1}^{N}\left(x_{i}-\bar{x}\right)^{2}\right)^{2}}-3
$$

And finally, an aggregation of the two statistical indicators is done by computing their arithmetic mean:

$$
A[X]=\frac{\sigma+\text { Kurt }}{2}
$$

An example of such an analysis carried out on a set of signals is shown in Fig. 7, where we can see the electromagnetic and acoustic sensors' processed outputs.
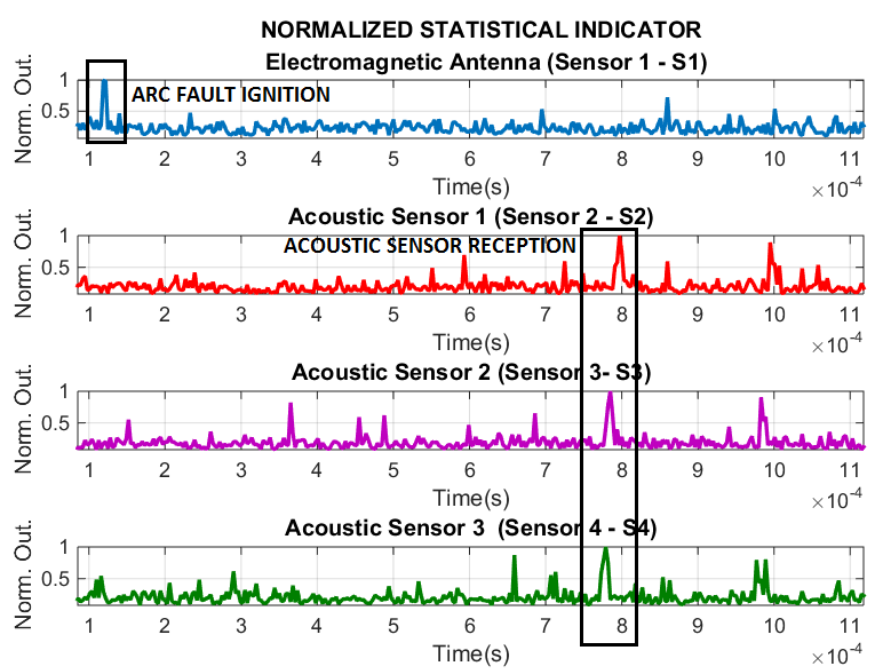

Fig. 7. Aggregate statistical indicator (normalized) example for a set of signals (one measurement).

\section{RESULTS}

The two methods were tested on arc fault sources located at different ranges, with the following spatial coordinates:

Config. 1: Xarc $=150 \mathrm{~cm}$, Yarc $=180 \mathrm{~cm}$, Zarc $=0 \mathrm{~cm}$;

Config. 2: Xarc $=180 \mathrm{~cm}$, Yarc $=180 \mathrm{~cm}$, Zarc $=0 \mathrm{~cm}$;

Config. 3: Xarc $=510 \mathrm{~cm}$, Yarc $=0 \mathrm{~cm}$, Zarc $=0 \mathrm{~cm}$.

The arc fault was placed at the same height as the centroid of the sensing system. The signals from Fig. 3 were obtained in this configuration and also, the processed signals in Fig. 7 were computed on the same signal set.

\section{A. Energy detector results}

For the configurations presented earlier, we have carried out five measurements, with the following mean results:

TABLE I. ENERGY DETECTOR: MEAN RESULTS \& ERRORS (\%).

\begin{tabular}{|c|c|c|c|c|}
\hline $\begin{array}{c}\text { Conf. } \\
\text { No. }\end{array}$ & \multicolumn{2}{|c|}{$\mathbf{X}_{\text {ARC }}$ Err.(\%) } & \multicolumn{2}{c|}{$\mathbf{Y}_{\text {ARC }}$ Err.(\%) } \\
\hline 1 & 154.3 & 2.9 & 187.9 & 4.4 \\
\hline 2 & 183.7 & 2.05 & 186.1 & 3.4 \\
\hline 3 & 519.6 & 1.88 & 2.4 & - \\
\hline
\end{tabular}

Table 1 shows us that there are reasonably low error percentages for this type of transient detection and TOA estimation. Normalized versions of the signals are presented in Fig. 8. 

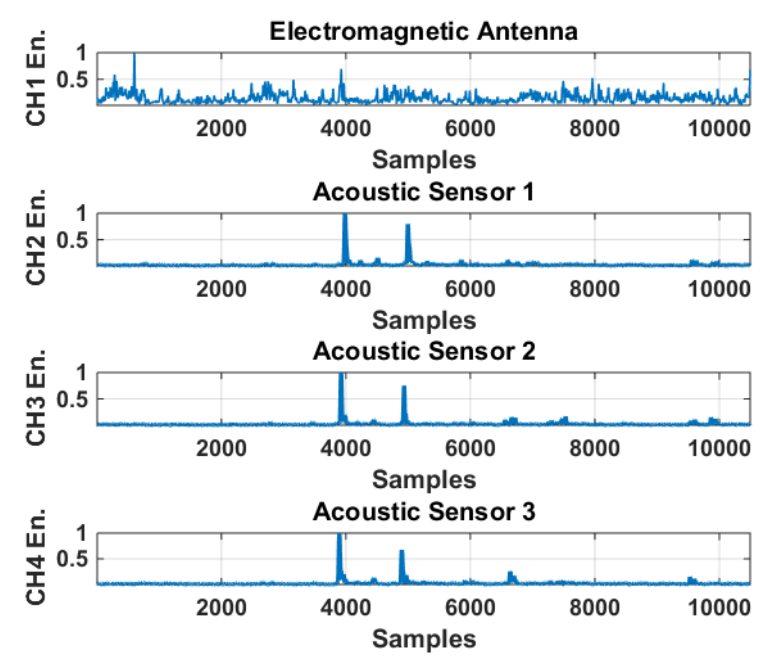

Fig. 8. Normalized windowed energy indicator (one measurement).

B. Sliding window kurtosis and standard deviation analysis 2:

The results of the second TOA estimation is given in Table

TABLE II. StATISTICAL ANALYSIS: Results \& ERRORS (\%).

\begin{tabular}{|c|c|c|c|c|}
\hline $\begin{array}{c}\text { Conf. } \\
\text { No. }\end{array}$ & \multicolumn{2}{|c|}{$\mathbf{X}_{\text {ARC }} /$ Err.(\%) } & \multicolumn{2}{c|}{$Y_{\text {ARC }} /$ Err.(\%) } \\
\hline 1 & 161.2 & 7.4 & 196.5 & 9.1 \\
\hline 2 & 187.2 & 4 & 196.8 & 9.3 \\
\hline 3 & 523.6 & 2.6 & 4.4 & - \\
\hline
\end{tabular}

The results are comparably worse than those obtained for the energy detector, but this may be caused by some internal sensitivities of the statistical approach, as we will explain in the concluding remarks.

\section{Statistical simulations based on the measurements}

For the energy detector, seeing as it offered the best performances (based on Tables I and II), we have carried out statistical simulations based on the results of the measurements. The procedure consisted in undertaking five measurements for each of the three configurations, and the following steps:

1. Compute means and standard deviations for each configuration.

2. Compute the corresponding normal distributions for each of the configurations.

3. Based on the normal distributions computed in the previous step, compute the survivor functions: Probability vs. Threshold.

The survivor functions are shown in Figures $9,10 \& 11$ and indicate how the probability of detection and probability of false alarm (noise) vary with the threshold and also with the distance between the arc fault source and the detection system.

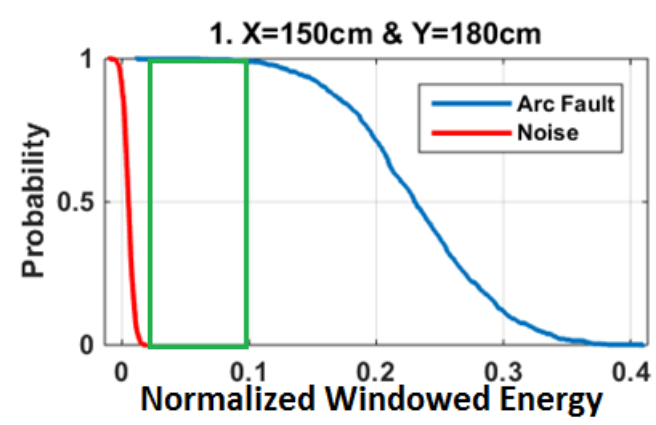

Fig. 9. Survivor function for the $1^{\text {st }}$ configuration: Probability of detection (Arc Fault - Blue) \& Probability of false alarm (Noise - Red) vs. Normalized Threshold, Range of Thresholds with $0 \%$ false alarms \& 100\% detection Green rectangle.

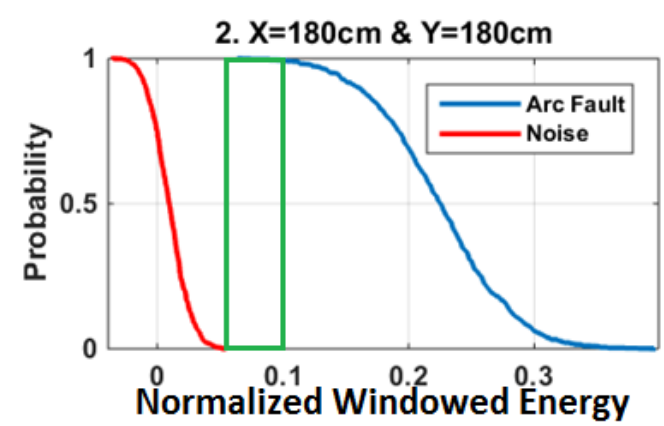

Fig. 10. Survivor function for the $2^{\text {nd }}$ configuration: Probability of detection (Arc Fault - Blue) \& Probability of false alarm (Noise - Red) vs. Normalized Threshold, Range of Thresholds with $0 \%$ false alarms \& $100 \%$ detection Green rectangle.

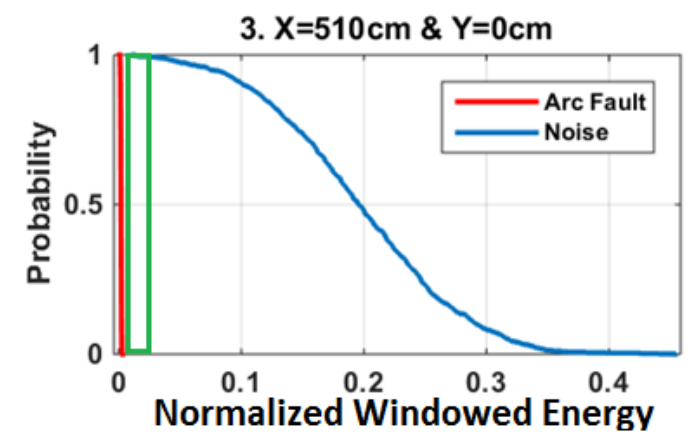

Fig. 11. Survivor function for the $3^{\text {rd }}$ configuration: Probability of detection (Arc Fault - Blue) \& Probability of false alarm (Noise - Red) vs. Normalized Threshold, Range of Thresholds with $0 \%$ false alarms \& 100\% detection Green rectangle.

Again, as can be seen from the statistical simulations carried out on the measurements, the farther away the arc fault source, the more difficult it is to find an adequate threshold that ensures $100 \%$ probability of detecting the arc faults and $0 \%$ probability of false alarm (of misclassifying noise as an arc fault).

\section{CONCLUSION}

It is clear that the better results were obtained by using the energy detector, as compared to those of the statistical analysis approach. 
For the sliding window statistical analysis there is always a compromise to be made between the length of the window $\rightarrow$ time resolution) and the overlap between windows $(\rightarrow$ "smoothness" of numerical indicator). The length of the window also influences greatly the validity of each statistical indicator, as a larger number of samples has the tendency to have more statistical relevance than a low-sample number window.

On the other hand, the energy detection scheme can be carried out even with a 1-sample overlap between integration windows, and have excellent time resolution and low SNR. A more complex detection scheme would be based on a known signal model for the arc fault generated pulses that would allow the use of a matched filter, that may even further improve the expected SNR [9].

The results obtained with the system and associated methods indicate the added value of further studying and modeling the spatial performances of our approach (maximal distance of detection), which will be carried out as future works.

More precisely, what is envisioned in the future for this project, is to carry out a detailed study on the statistical performances (probability of detection and probability of false alarm) with regards to the position of the arc fault source, in order to obtain a 3D mapping of the expected performances. The energy detector still stands as the go-to method, but other approaches may be investigated in order to determine the Time-of-Arrival.

The hardware platform that was designed (the multisensing system), in conjunction with the developed methods and its associated results, show the extremely promising nature of the electromagnetic-acoustic detection approach.

\section{ACKNOWLEDGMENT}

This work has been supported by the Institut Carnot "Energies du Futur" grant, Dema'loc.

\section{REFERENCES}

[1] J. Saulnier, J. Ghouili, "Detection of Electrical Arc Faults in a Distribution Network", IEEE CCECE, pp. 571 - 574, 2007.

[2] J. Flicker, J. Johnson, "Electrical simulations of series and parallel PV arcfaults", IEEE PVSC, pp. 3165 - 3172, 2013.

[3] J. Johnson et al., "Photovoltaic DC Arc Fault Detector testing at Sandia National Laboratories", IEEE PVSC, 003614-003619 (2011).

[4] http://tgalsolar.com/2012/09/05/safety-ac-dc-choice/, "Safety, AC v. DC your choice", 2012.

[5] A. Digulescu, M. Paun, C. Vasile, T. Petrut, D. Deacu, C. Ioana, R. Tamas, "Electrical arc surveillance and localization system based on advanced signal processing techniques", IEEE ENERGYCON, pp. 426-430, 2014.

[6] A. Digulescu, I. Candel, J. Dahmani, D. Deacu, C. Ioana, V. Gabriel, "Electric arc locator in photovoltaic power systems using advanced signal processing techniques", IEEE ELMAR, pp. 129-132, 2013.

[7] A. Digulescu,T. Petrut,C. Bernarnd, I. Candel, C. Ioana, A. Serbanescu, "Advanced signal processing techniques for detection and localization of electrical arcs", IEEE COMM, pp. 1-4, 2014.

[8] H. Urkowitz, " Energy detection of unknown deterministic signals", Proceedings of the IEEE, vol. 55, pp. 523-531, 1967.

[9] T. Misaridis, J. A. Jensen, "Use of modulated excitation signals in medical ultrasound. Part I: basic concepts and expected benefits", IEEE Transactions on Ultrasonics, Ferroelectronics and Frequency Control, vol. 52, pp. 177-191, 2005.

[10] S. Atapattu, C. Tellambura, H. Jiang, "Energy Detection for Spectrum Sensing in Cognitive Radio", Springer, pp. 11-27, 2014. 